\title{
A Model of the Role of Cholesterol in the Development of Alzheimer's Disease
}

\author{
Gizelle Kupac Vianna ${ }^{1}$, Artur E. Reis ${ }^{1}$, Luís Alfredo V. de Carvalho ${ }^{1}$, \\ and Roseli S. Wedemann ${ }^{2}$ \\ 1 COPPE - Universidade Federal do Rio de Janeiro, \\ Prog. Eng. de Sistemas e Computação, P.O. Box 68511, 21945-970, Rio de Janeiro, RJ, Brazil \\ gkupac@gmail.com, artur_reis@yahoo.com.br, LuisAlfredo@ufrj.br \\ ${ }^{2}$ Instituto de Matemática e Estatística, Universidade do Estado do Rio de Janeiro \\ R. São Francisco Xavier, 524, 20550-013, Rio de Janeiro, RJ, Brazil \\ roseli@ime.uerj.br
}

\begin{abstract}
We present a mathematical-computational model of the development of Alzheimer's disease, based on the assumption that cholesterol plays a key role in the formation of neuropathological lesions that characterize the disease: the senile amyloid plaques and neurofibrillary tangles. The final model, conceived as a system of equations, was simulated by a computer program.
\end{abstract}

\section{Introduction}

We propose a mathematical-computational model, that represents the physiological alterations resulting from Alzheimer's Disease (AD) [9]. It is difficult to achieve a good understanding of this pathology, since it is generated by a combination of many complex and highly connected processes. The model thus aims at facilitating the comprehension of $\mathrm{AD}$, which is associated with an adaptative biological complex system. We know of no other similar attempt to achieve such a model, in the specialized literature.

$\mathrm{AD}$ affects almost $7 \%$ of the population over 65 years old and up to $40 \%$ of people over 80 years of age, and it is estimated that it is responsible for almost $70 \%$ of all senile dementias. AD consists of a degenerative process, characterized by the occurrence of a series of abnormalities in the brain, selectively affecting neurons of specific regions, such as the cortex and the hippocampus. Microscopic exams reveal a great amount of two physical alterations that characterize AD and distinguish it from other diseases: extracellular senile amyloid plaques ( $S A P$ 's) and intracellular neurofibrillary tangles ( $N T$ 's). $S A P$ 's consist of deposits of amyloid $\beta$-peptide $(A \beta)$ surrounded by distrofic axons and inflammation. $N T$ 's contain paired helical filaments, composed of hyperphosphorylated forms of Tau protein [2]. Such alterations hinder the functioning of sinapses and the feasibility of neurons, leading to neuronal loss.

Age is the most relevant risk factor for $\mathrm{AD}$, as many physical alterations take place in the brain as an individual grows older. The theory of oxidative stress conceives aging as a result of the accumulation of damages to the tissues caused by free radicals [8]. Some epidemiologic studies suggest an influence displayed by high plasmatic cholesterol levels in the development of AD [5]. The disease also shows some genetic components. 


\section{A Computational Model and Simulations}

There is uncertainty regarding the relationship between levels of cholesterol in the plasma and in the central nervous system $(C N S)$, in patients with AD. This is due to the fact that plasma cholesterol and brain cholesterol are separated by the blood-brain barrier $(B B B)$, that controls the exchange of substances between fluids of the brain and blood. However, there is evidence that the level of cholesterol in the frontal cortex demonstrates a significant increase when plasma cholesterol increases [1].

In [9], we have presented a mathematical model which was simulated by a computer program for the main mechanisms in $\mathrm{AD}$ that are influenced by the presence of cholesterol in the CNS, as proposed in the literature. The model consists of a set of linear equations and linear differential equations that model the concentration of substances involved in the metabolism of cholesterol, the formation of senile amyloid plaques and in the formation of neurofibrilary tangles, all of which cause neuronal loss [1, 2, 3, 4, 5, 6, 7, 8]. We consider that the level of neuronal loss will characterize $\mathrm{AD}$, since it relates to the cerebral atrophy that occurs in the disease.

We used Euler's method to solve the model equations. One integration step, representing time, corresponds to 8 hours and the simulation of 100 years requires approximately 100,000 steps. A basal cholesterol diet corresponds to a daily consumption of $300 \mathrm{mg}$ and a high cholesterol diet to a daily consumption of $600 \mathrm{mg}$. In Fig. 1] we show the production levels of $S A P$ 's and $N T$ 's from a simulation of daily ingestion of $600 \mathrm{mg}$ of cholesterol.

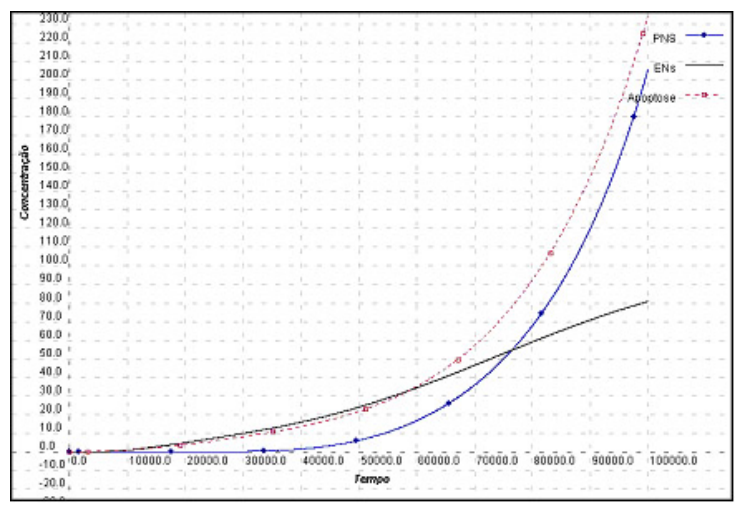

Fig. 1. $[S A P](\mathrm{PNS})$ and $[N T](\mathrm{ENs})$, for $600 \mathrm{mg}$ of cholesterol and $A \beta$ aggregation

Murray [4] hypothesized that, if the process of aggregation of $A \beta$ could be suspended, the disease would not evolve. We tested the system's behavior when the aggregation process of $A \beta$ is inhibited. The results in Fig. 2 are coherent with the hypothesis and we observe that, when the aggregation process of $A \beta$ is inhibited, suppression of the inflammatory process occurs and the formation of $S A P$ 's is inhibited. We notice a slight decrease in the formation rate of $N T$ 's. As $S A P$ 's were no longer formed, 


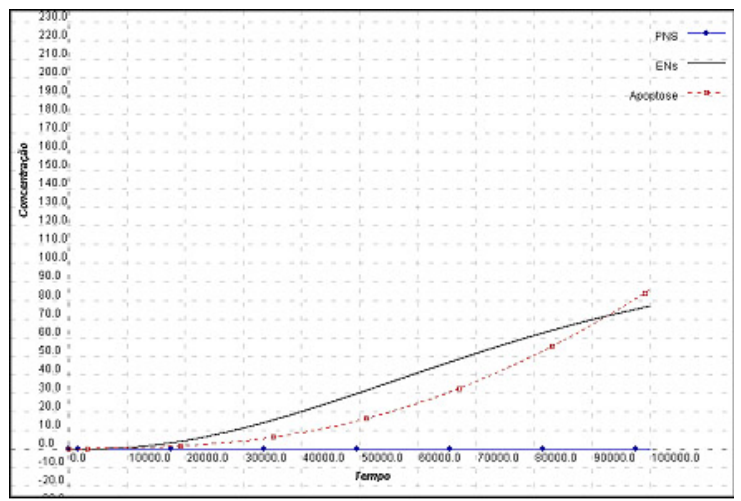

Fig. 2. $[S A P]$ and $[N T]$, for $600 \mathrm{mg}$ of cholesterol and inhibition of $A \beta$ aggregation

there will be no disturbance in the functioning of the $B B B$ by these structures and the increase of efflux of cholesterol is avoided. Changes in the distribution of cholesterol in the neuronal membrane will occur much more slowly and the production of $A \beta$ is reduced.

There are some positive feedbacks in the system that may aggravate AD. For example, $A \beta$ and $S A P$ 's initiate an inflammatory process in the brain, which aggravates the AD development process. We inhibited the occurrence of inflammation and observed a keen reduction in the development of neuropathological alterations, with daily ingestion of $600 \mathrm{mg}$, as seen by comparing Figures 1 and 3 .

In Niemann-Pick's disease, $N T$ 's identical to those of AD were found, but no $S A P$ 's. Since individuals with this disease usually die much younger than AD patients, we conclude that $N T$ 's occur earlier than $S A P$ 's. Our simulations confirm this phenomenon as shown in Fig. 11 since the formation of $N T$ 's happens almost 25 years before $S A P$ 's.

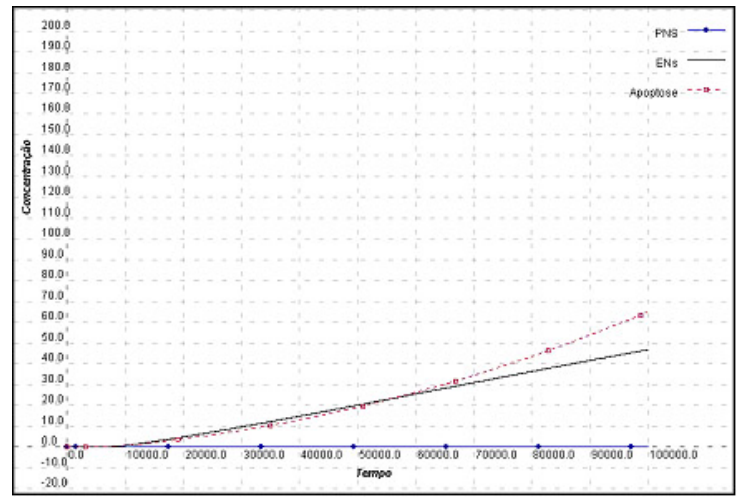

Fig. 3. Neuropathological alterations for $600 \mathrm{mg}$ cholesterol, without inflammation 


\section{Conclusions}

Results from the literature and from our simulations suggest that, despite the brain's resistance to dietary lipid composition, chronic consumption of cholesterol may alter the functioning of certain cerebral proteins and even the structure of neurons. This happens because cholesterol alters the composition and certain properties of the neuronal membrane. One consequence of these alterations is the increase of $A \beta$ production and aggregation, simultaneous with a reduction of its degradation rate. These factors contribute to the increase of toxic $A \beta$ formation, leading to formation of $S A P$ 's which cause the generation of free radicals, amplifying oxidative stress $O S . O S$ then oxidates the synaptic plasmatic membranes, creating a positive feedback over $A \beta$ production. Finally, $O S$ and insoluble $A \beta$ trigger Tau protein phosphorylation and the neuronal structure is destabilized, generating $N T$ 's. Inflammation also plays an important role in the development of $\mathrm{AD}$ and its inhibition resulted in a sharp deceleration of the neurodegenerative process.

Acknowledgements. This research was developed with grants from the brazilian National Research Council (CNPq), the Rio de Janeiro State Research Foundation (FAPERJ) and the brazilian agency which funds graduate studies (CAPES).

\section{References}

1. Howland, D. et al.: Modulation of Secreted $\beta$-Amyloid Precursor Protein and Amyloid $\beta$ Peptide in Brain by Cholesterol. The J. of Biological Chemistry, 273 No. 26. (1998) 1657616582

2. Illenberger, S. et al.: The Endogenous and Cell Cycle-Dependent Phosphorylation of Tau Protein in Living Cells: Implications for Alzheimer's Disease, Molecular Biology of the Cell, 9 (1998) 1495-1512

3. Ji, S.R., Wu, Y., Sui, S.F.: Cholesterol is an Important Factor Affecting the Membrane Insertion of $\beta$-Amyloid Peptide (A $\beta$-40), which May Potentially Inhibit the Fibril Formation. The J. of Biological Chem., 277 No. 8. (Feb 22, 2001) 6273-6279

4. Murray, R.K.: The Biochemical Basis of Some Neuropsychiatry Disorders. In: Murray, R.K., Granner, D.K., Mayers P.A. et al. (eds.): Harpers Biochemistry, Int. Edition, (23 ed.). PrenticeHall (1993) 750-752.

5. Pappolla, M.A., Smith, M.A., et al.: Cholesterol, Oxidative Stress, and Alzheimer's Disease: Expanding the Horizons of Pathogenesis. In: Smith, M.A., Perry, G. (eds.): Serial Review: Causes and Consequences of Oxidative Stress in Alzheimer's Disease. Free Radical Biology and Medicine, Vol. 33, no. 2., Elsevier (2002) 173-181

6. Rissman, R.A., Poon, W.W., Jones, M.B. et al.: Caspase-cleavage of Tau is an early event in Alzheimer's disease tangle pathology. The J. of Clinical Investigation, 114 No. 1, (2004) $121-130$

7. Selkoe, D.J.: Toward a Comprehensive Theory for Alzheimer's Disease. Hypothesis: Alzheimer's Disease is Caused by the Cerebral Accumulation and Cytotoxicity of Amyloid $\beta$-Protein. Annals of the New York Academy of Science, 924 (2000) 17-25

8. Wood, W.G. et al.: Brain Membrane Cholesterol Domains, Aging and Amyloid Beta-Peptides. Neurobiology of Aging, No. 23 (2002) p.685-694

9. Vianna, G.K.: Um Modelo Neurocomputacional do Papel do Colesterol no Desenvolvimento da Doença de Alzheimer. Ph.D. Dissertation, Prog. Eng. de Sistemas e Computação, Universidade Federal do Rio de Janeiro, Brazil, (2005) (in Portuguese). 